\title{
Supporting Information for: Xenon on Graphene/Pt(111) at Low Temperature: Influence of the Metal Support
}

\author{
Mariano D. Jiménez-Sánchez, ${ }^{\mathrm{a},{ }^{*}}$ Alba Payés-Playà, ${ }^{\mathrm{a}}$ Bruno de la Torre, ${ }^{\mathrm{a}, \mathrm{b}}$ Nicoleta \\ Nicoara $^{c}$ and José M. Gómez-Rodríguez ${ }^{\mathrm{a}, \mathrm{d}, \mathrm{e}}$, * \\ ${ }^{a}$ Departamento de Física de la Materia Condensada \\ Universidad Autónoma de Madrid \\ E-28049 Madrid, Spain \\ ${ }^{b}$ Present address: Palacky Univ, Reg Ctr Adv Technol \& Mat, \\ Olomouc 78371, Czech Republic \\ ${ }^{c}$ International Iberian Nanotechnology Laboratory (INL) \\ 4715-330 Braga, Portugal \\ ${ }^{\mathrm{d}}$ Instituto Nicolás Cabrera \\ Universidad Autónoma de Madrid \\ E-28049 Madrid, Spain \\ ${ }^{\mathrm{e}}$ Condensed Matter Physics Center (IFIMAC) \\ Universidad Autónoma de Madrid \\ E-28049 Madrid, Spain
}

* Corresponding authors: Departamento de Física de la Materia Condensada,
Universidad Autónoma de Madrid, E-28049, Madrid, Spain
E-mail adresses: josem.gomez@uam.es (José M. Gómez-Rodríguez);
marianod.jimenez@uam.es (Mariano D. Jiménez-Sánchez). 


\section{Xe layer nanomanipulation by STM}

We have been able to nanomanipulate the Xe overlayer with the STM. In particular, we have formed single Xe vacancies by picking single Xe atoms with the STM tip by means of slight tip-sample contacts, in a similar way as that used, since the seminal work of Eigler and Schweizer ${ }^{1}$ by other authors with Xe adatoms on metal surfaces ${ }^{2-3}$. Figure S1 shows an example of such nanomanipulations where a single Xe adatom was extracted from the Xe overlayer on $\mathrm{G} / \mathrm{Pt}(111)$.

a)

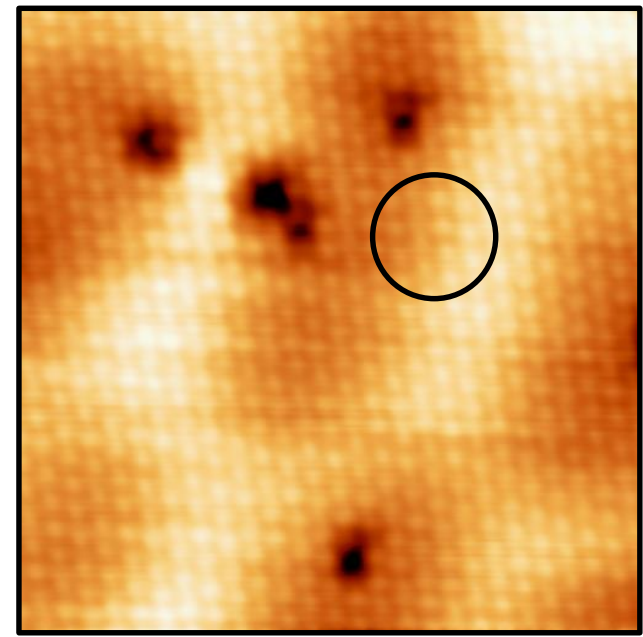

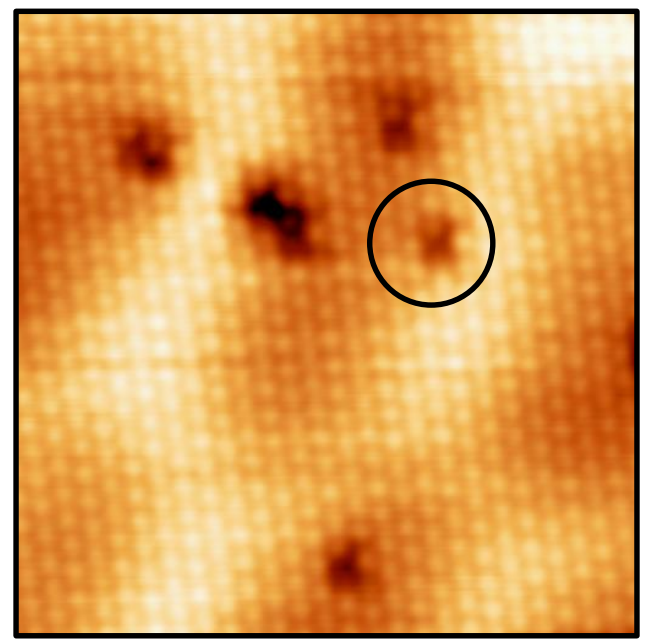

)

Fig. S1: STM nanomanipulation leading to the extraction of a single Xe atom from a Xe single layer island on the G/Pt(111) surface. The tip was stopped on a selected spot and then approached by $0.7 \mathrm{~nm}$ towards the sample (with the feedback loop opened). (a) surface before and (b) after the extraction and formation of a single Xe vacancy (see black circle drawn on both images). Size: $10.5 \mathrm{~nm} \times 10.5 \mathrm{~nm}$; Sample bias voltage: $-2.5 \mathrm{~V}$; Tunneling current: $0.05 \mathrm{nA}$; Temperature: $5 \mathrm{~K}$. 


\section{Identification of graphene moirés under the Xe overlayer by STM}

\section{nanomanipulation}

In some cases, it has been possible to remove large areas of the Xe overlayer without deteriorating the STM tip, by scanning at low tunnel resistance. In these cases, that nanomanipulation has helped to identify the underlying moiré by an alternative method to the one used in the main manuscript (imaging the graphene terraces close to Xe islands). Fig. S2 shows an example of this procedure based on STM nanomanipulation.

a)

b)

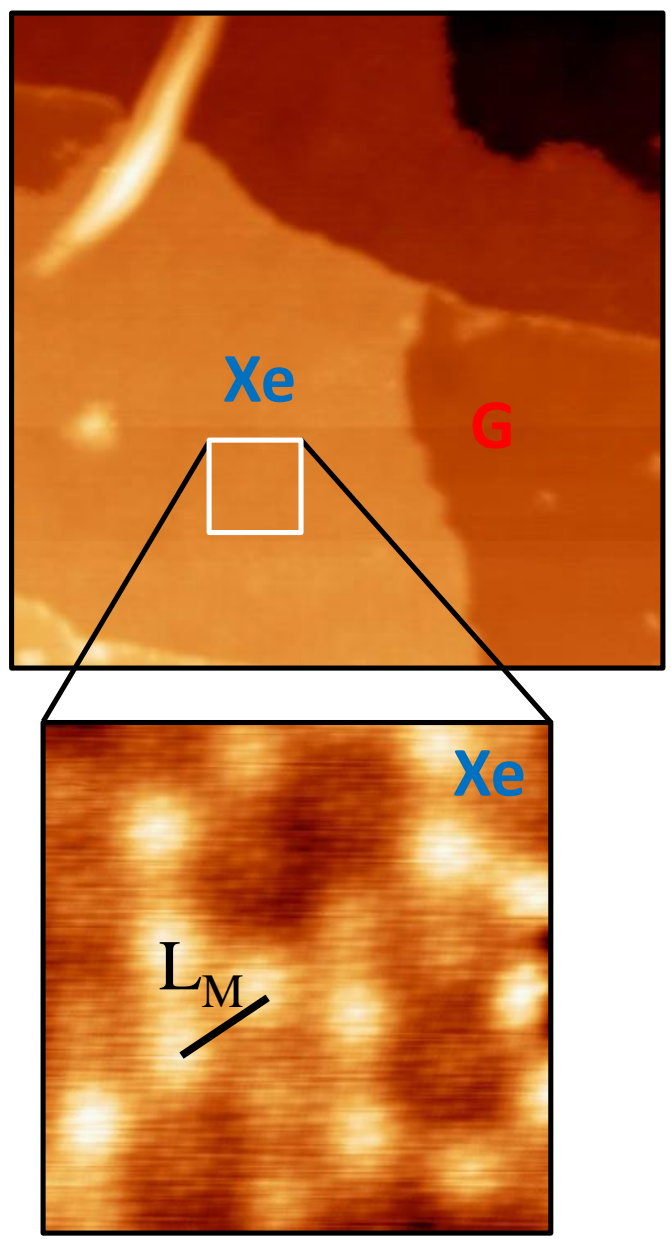

c)

d)

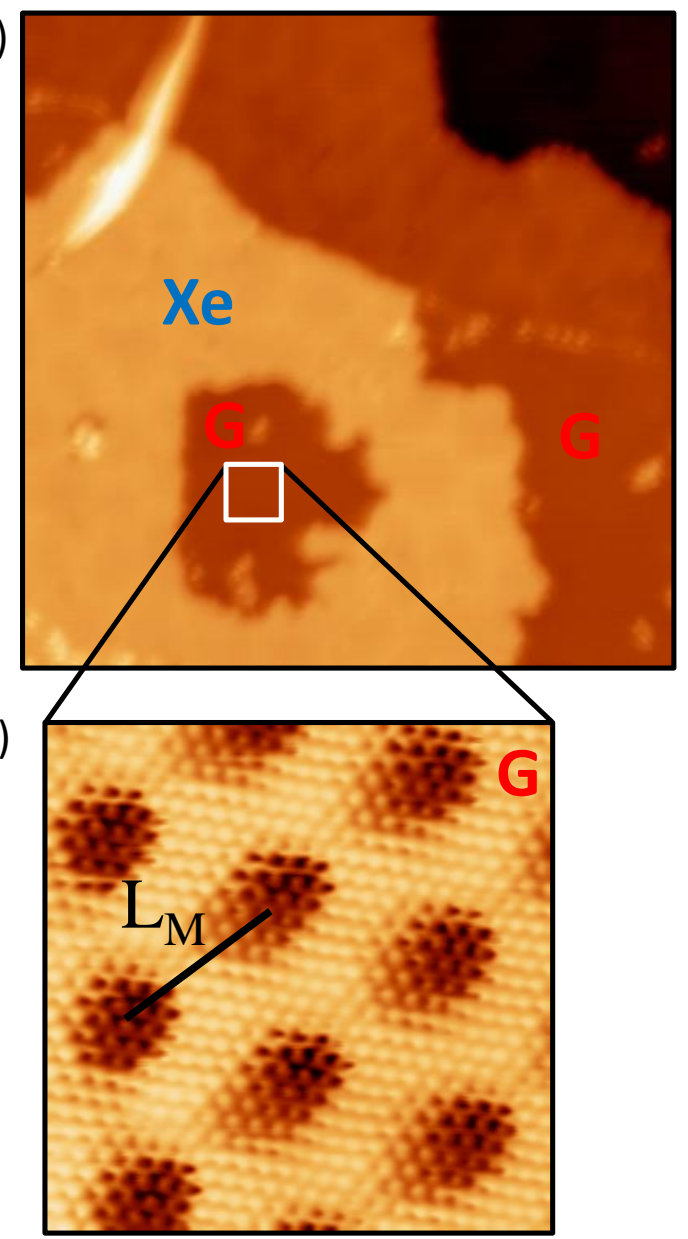

Fig. S2: STM nanomanipulation of a Xe single layer island on $\mathrm{G} / \mathrm{Pt}(111)$ leading to the removal of a large portion of the Xe island. (a) shows a $70 \mathrm{~nm}$ $\times 70 \mathrm{~nm}$ STM image of a region before its manipulation. A zoom-in $(10 \mathrm{~nm}$ $\times 10 \mathrm{~nm}$ ) measured in the Xe island is displayed in (b). Three periodicities 
can be well resolved: $L_{I C}>L_{M}(\sim 2.1 \mathrm{~nm})>L_{X e}$, corresponding to the incommensurate honeycomb, the underlying graphene moiré and the atomic Xe lattices. (c) corresponds to the same area in (a) and displays the result of the removal of a large central part of the Xe island by scanning locally this area at low tunnel resistance (sample bias: $-19 \mathrm{mV}$; tunneling current: 6.5 $\mathrm{nA})$. A zoom-in $(6 \mathrm{~nm} \times 6 \mathrm{~nm})$ measured in the manipulated zone is shown in (d). A G/Pt(111) clean surface is observed, presenting the periodicity of a large moire $\left(L_{M}\right)$, exactly the same as that measured in (b), and the atomic periodicity of graphene. The temperature on all the measurements was $5 \mathrm{~K}$.

\section{Contrast inversion in STM images}

We have detected contrast inversions in the STM images of the incommensurate phase of $\mathrm{Xe}$ on $\mathrm{G} / \mathrm{Pt}(111)$ by changing the sample bias voltage. Contrast inversions were also detected by Grimm et al. in the case of $\mathrm{Xe} / \mathrm{HOPG}^{4}$.

In fig. $\mathrm{S} 3$ we show the inversion of contrast when changing from a sample bias of -2.4 $\mathrm{V}$ (the hexagonal commensurate domains are observed as dark areas while the domain walls are observed as light areas) to a sample bias of $+3.5 \mathrm{~V}$ (the hexagonal commensurate domains are observed as light areas while the domain walls are as dark regions). 
a)

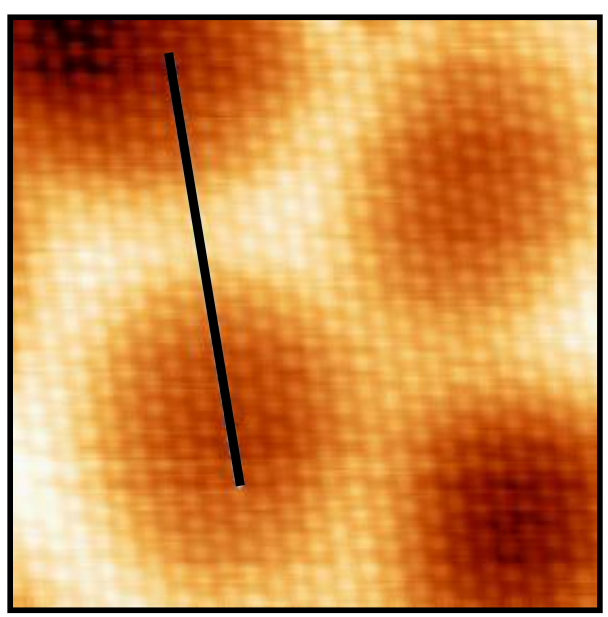

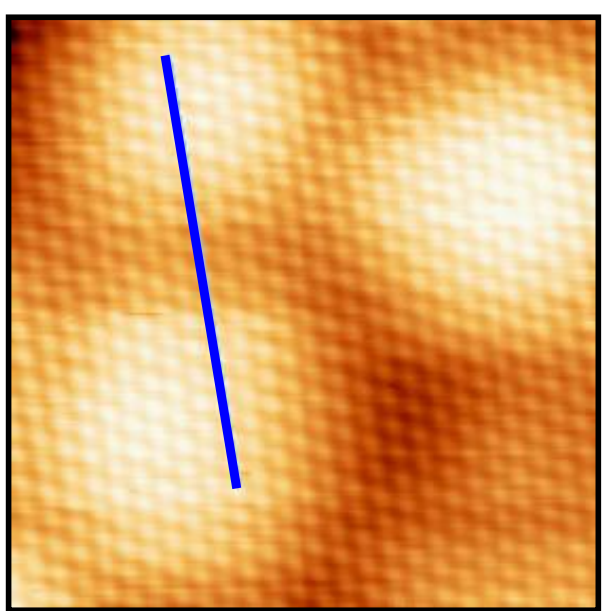

b)

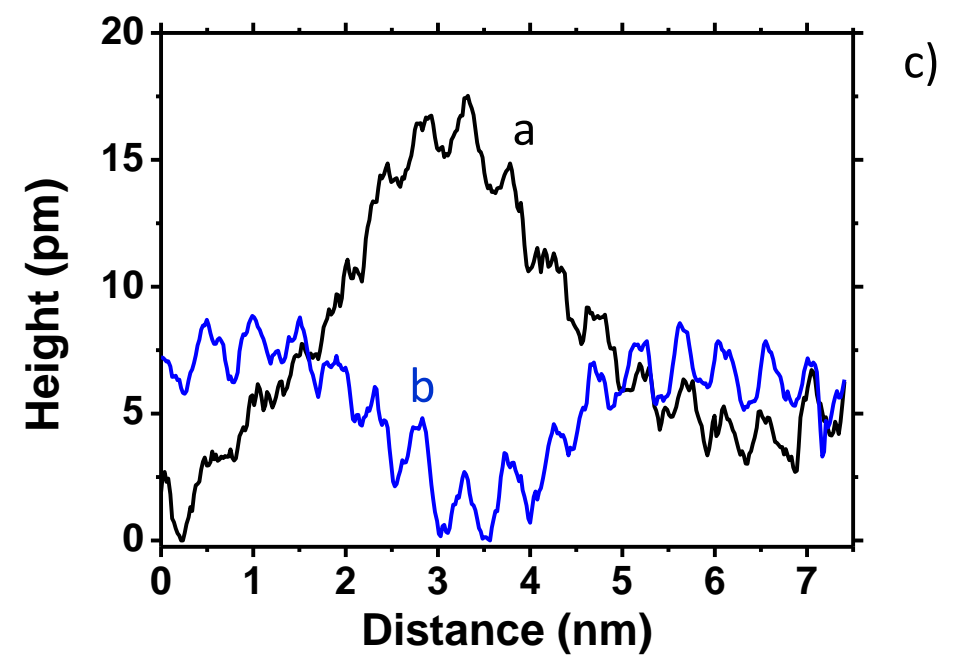

Fig. S3: Contrast inversion of the STM images on Xe on G/Pt(111) by the change of the sample bias voltage. The same image area $(10 \mathrm{~nm} \times 10 \mathrm{~nm})$ is scanned in (a) and (b) but the sample bias voltage is different: $-2.4 \mathrm{~V}$ (a) and $+3.5 \mathrm{~V}$ (b). The tunneling current is $0.090 \mathrm{nA}$ for both images and the measurement temperature is $5 \mathrm{~K}$. (c) shows the comparative profiles of the apparent height for (a) (black curve) and (b) (blue curve).

\section{Xe-G/Pt(111) imaging at $40-70 \mathrm{~K}$}

By means of a home-made variable temperature (VT)-STM ${ }^{5}$ installed in another UHV set-up, it has been possible to image the $\mathrm{Xe}$ on $\mathrm{G} / \mathrm{Pt}(111)$ system also at much higher temperatures $(40-70 \mathrm{~K})$ than those used in the main manuscript $(5 \mathrm{~K})$. At such high 
temperatures the Xe overlayer is quite mobile, making stable STM imaging difficult. However, the two kind of regions described in the main manuscript and measured at 5 $\mathrm{K}, 2 \mathrm{LR}$, i.e. those presenting only two periodicities, and $3 \mathrm{LR}$, those presenting three periodicities, being one of them related to the underlying $\mathrm{G} / \mathrm{Pt}(111)$ large and corrugated moiré, are also observed at high temperature, as shown in fig. S4.

a)

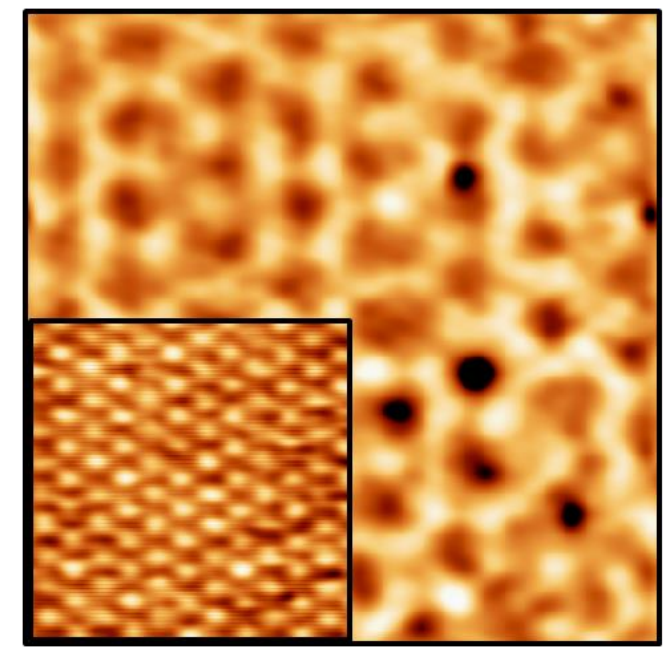

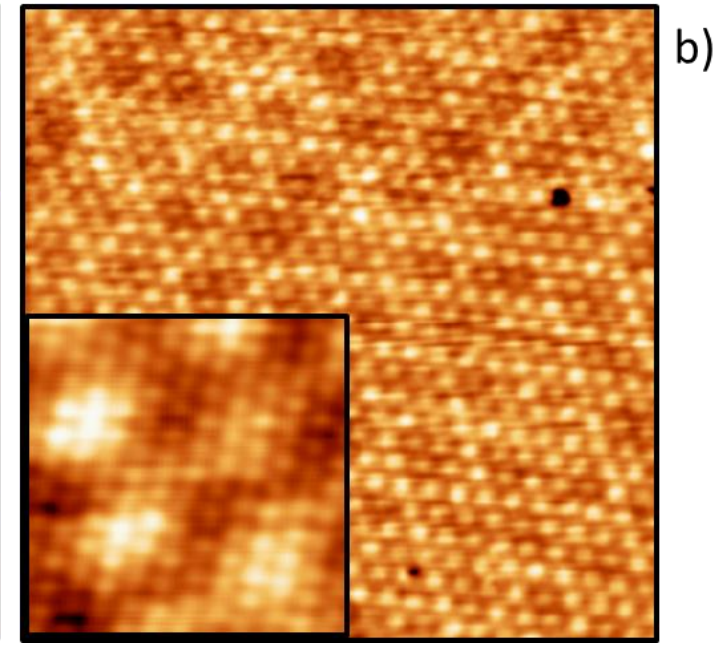

Fig. S4: STM images measured with a VT-STM at temperatures much higher than the rest of the present work. (a) presents a 2LR-type island (50 $\mathrm{nm} \times 50 \mathrm{~nm}) ;$ the large honeycomb lattice corresponding to the incommensurate phase can be observed. The inset is a zoom-in $(4.5 \mathrm{~nm} \times$ $4.5 \mathrm{~nm}$ ) in a 2LR-type island showing the Xe atomic resolution. (b) presents a 3LR-type island $(50 \mathrm{~nm} \times 50 \mathrm{~nm})$, where the honeycomb lattice and the periodicity corresponding to the high corrugated moiré can be observed. A zoom-in is shown in the inset $(4.5 \mathrm{~nm} \times 4.5 \mathrm{~nm})$, where the moiré-type periodicity and the Xe atomic resolution can be observed. The tunneling parameters and temperatures are: (a) main image: $\mathrm{V}_{\mathrm{s}}=-3.8 \mathrm{~V} ; \mathrm{I}_{\mathrm{t}}=0.005$ nA; zoom-in image: $\mathrm{V}_{\mathrm{s}}=1.4 \mathrm{~V} ; \mathrm{I}_{\mathrm{t}}=0.3 \mathrm{nA} ; \mathrm{T}=40 \mathrm{~K}$; (b) main image: $\mathrm{V}_{\mathrm{s}}=$ $-3.0 \mathrm{~V} ; \mathrm{I}_{\mathrm{t}}=0.095 \mathrm{nA}$; zoom-in image: $\mathrm{V}_{\mathrm{s}}=-3.0 \mathrm{~V} ; \mathrm{I}_{\mathrm{t}}=0.095 \mathrm{nA} ; \mathrm{T}=68 \mathrm{~K}$ 


\section{References}

1. Eigler, D. M.; Schweizer, E. K. Positioning Single Atoms with a Scanning Tunneling Microscope. Nature 1990, 344, 524-526.

2. Mohn, F.; Schuler, B.; Gross, L.; Meyer, G. Different Tips for High-Resolution Atomic Force Microscopy and Scanning Tunneling Microscopy of Single Molecules. Appl. Phys. Lett. 2013, 102, 073109.

3. Kichin, G.; Weiss, C.; Wagner, C.; Tautz, F. S.; Temirov, R. Single Molecule and Single Atom Sensors for Atomic Resolution Imaging of Chemically Complex Surfaces. J. Am. Chem. Soc. 2011, 133, 16847-16851.

4. Grimm, B.; Hovel, H.; Pollmann, M.; Reihl, B. Physisorbed Rare-Gas Monolayers: Evidence for Domain-Wall Tilting. Phys Rev Lett 1999, 83, 991-994.

5. Custance, O.; Brochard, S.; Brihuega, I.; Artacho, E.; Soler, J. M.; Baro, A. M.; Gomez-Rodriguez, J. M. Single Adatom Adsorption and Diffusion on Si(111)-(7x7) Surfaces: Scanning Tunneling Microscopy and First-Principles Calculations. Phys Rev B 2003, 67, 235410. 